VII. UV OBSERVATIONS AND MASS LOSS 


\section{J.M. Marlborough}

Astronomy Centre, The University of Sussex, Falmer, U.K. and

Department of Astronomy, The University of Western Ontario, London, Ontario, Canada.

\section{INTRODUCTION}

Although the first ultraviolet (UV) observation of an astronomical source was obtained in 1946, the first UV observations of Be stars were not obtained until 1964. In this review of UV data covering the period since 1964, the term Be star will be assumed to include Oe stars as well (Frost and Conti, 1976). An earlier review of this subject is by Heap (1976).

Ultraviolet astronomy refers generally to the wavelength interval $912<\lambda \leq 3000 \mathrm{~A}$, these 1 imits arising respectively from the absorbing properties of the interstellar gas and the earth's atmosphere. This wavelength range is sometimes further divided into the near UV, $2000<\lambda<3000 \mathrm{~A}$, and the far UV, $912 \leq \lambda \leq 2000 \mathrm{~A}$. In the near UV balloons can Eransport detectors to sufficiēntly high altitude at which a significant residual signal remains. For the far UV however rockets and satellites are the only practical vehicules. Observations of $\mathrm{Be}$ stars have been obtained in a variety of space experiments. Some of the characteristics of these are given in Table 1.

The UV spectra of Be stars resemble closely those of B stars, although there are important quantitative differences. Qualitatively the two obvious differences between the optical and UV for Be stars are the lack of emission lines and the high density of absorption lines. Although infrequently present, emission lines have been noted or suspected at N V $\lambda 1240$ (Morton, 1976; Marlborough and Snow, 1980), C IV $\lambda 1550$ (Bohlin, 1970), Mg II $\lambda 2800$ (Kondo et a1., 1975) and Si IV $\lambda 1400$ (Marlborough et al., 1978). There appears to be no correlation between the presence of UV emission lines and the strength of Balmer emission lines, even though at optical wavelengths when the latter are strong, emission lines of Fe II are present (McLaughlin, 1961). Absorption lines can be either photospheric, circumstellar, or interstellar. Separation of photospheric and interstellar lines is straightforward, especially if $v$ sin $i$ is moderate or large. Distinction between circumstellar and interstellar lines is also possible, 


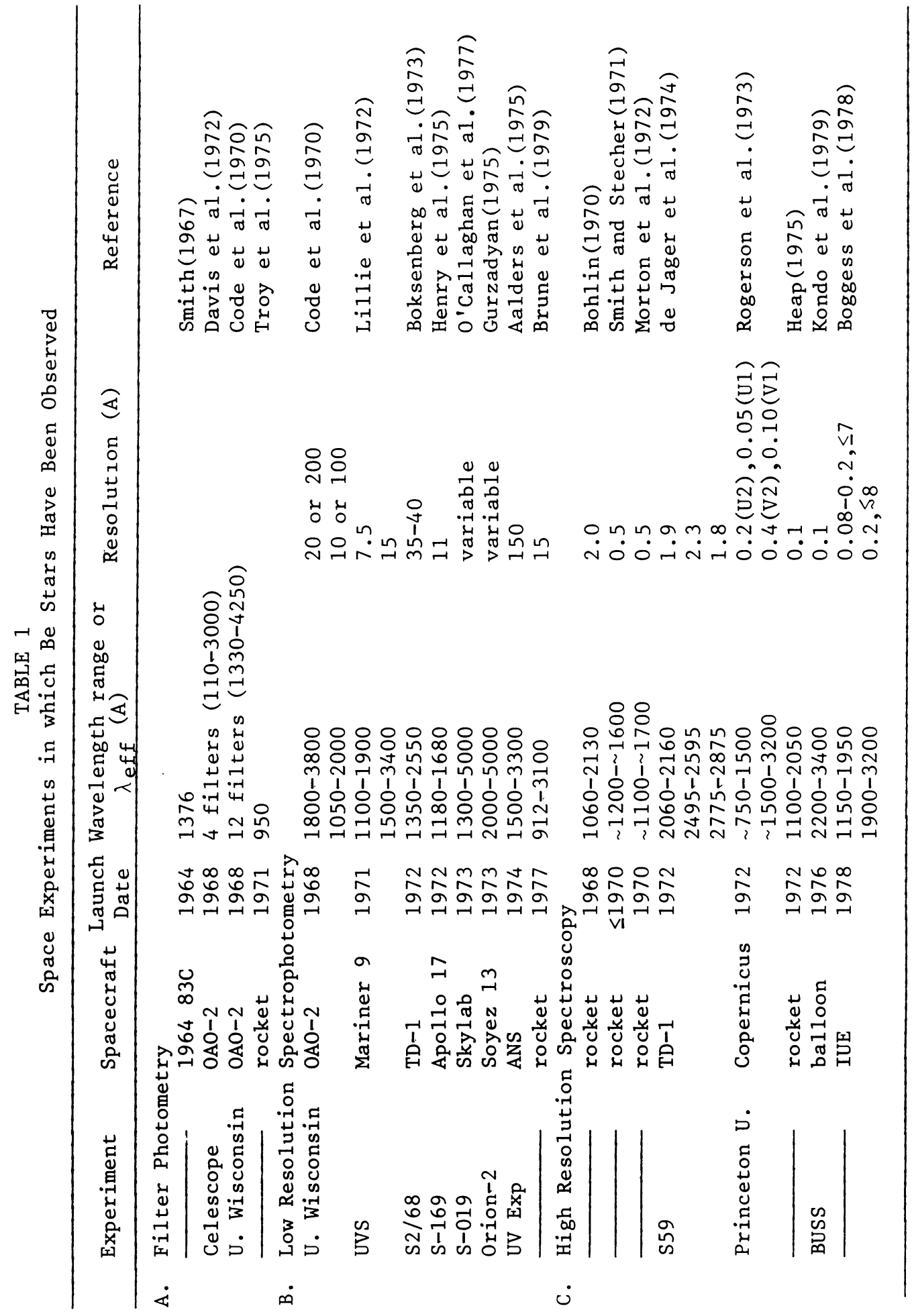


although more difficult, because circumstellar lines may be narrower than photospheric ones. The standard approach for study of optical spectra can be used to distinguish between photospheric and circumstellar lines.

As expected the UV spectra of B stars were found to contain a very large number of absorption lines (Burger and van der Hucht, 1976; Rogerson and Upson, 1977). This high line density leads to a number of problems. Identification of spectral features on low resolution spectra is complicated because all features are blends; assignment of a single contributor to a particular feature may be very misleading if not totally incorrect. In principle this difficulty can be reduced by obtaining spectra of higher resolution. However as Kurucz (1974) has demonstrated rapid rotation leads to an intrinsic blending which cannot be overcome by higher instrumental resolution. Finally both Kurucz (1974) and Peytremann (1975) have emphasized that the large number of lines in the UV together with limited resolution, either instrumental and/or intrinsic, make the location of the continuum very uncertain.

\section{THE CONTINUOUS ENERGY DISTRIBUTION}

Observations of the continuous energy distribution in the UV for $\mathrm{Be}$ and $B$ stars are very important for meaningful tests of the predictions of model atmospheres and of the effects of rotation on energy distributions, and also for better estimates of $\mathrm{T}_{\text {eff }}$ and bolometric corrections. Furthermore the amount and distribution of radiation in the Lyman continuum is an important input quantity in constructing models of the circumstellar envelope (CE) of Be stars.

Beeckmans and Hubert-Delplace (1980) have determined the ratio of the strength of the UV continuum $(1650<\lambda<2500 \mathrm{~A})$ to that of the Paschen continuum in 63 stars using $5276 \overline{8}$ spectra. For each Be star they compared this ratio with the expected value for a normal star of the same spectral type, the latter being obtained from analysis of spectra of 200 normal B stars. They conclude that: Be stars earlier than $B 5$ have less UV flux at $\lambda 2100$, relative to the optical $V$ band, than do normal B stars; Be stars later than B5 may also have this flux deficit but the conclusion is less certain; and the magnitude of this flux deficiency is correlated with optical emission line strength, infrared excess and the presence of a shell. An earlier study by Briot (1976) found essentially no difference in UV flux of Be stars relative to $B$ stars; her conclusions however are suspect because of the method used to correct for interstellar extinction.

At the present time this UV flux deficiency may be explained by the combined effects of line blocking together with hydrogen bound-free absorption, especially in stars with conspicuous shells. Poeckert and Marlborough (1978b) have shown that the predicted strength of both Balmer emission lines and the infrared continuum for ad hoc models of 
the $C E$ increase with increasing envelope density. Snow et al. (1979) discovered UV Fe III absorption lines in a number of Be stars whose optical spectra showed no evidence for shell lines. Marlborough and Snow (1980) noted that significant absorption by UV Fe III was evident in 59 Cyg at a time when the optical spectrum was expected to have strong Balmer emission but at most only weak shell 1ines. Finally Beeckmans (1976) showed that the UV continuum of 59 Cyg was much fainter, when the optical spectrum displayed strong shell lines, than at times when no optical she11 lines were present.

Various groups have obtained absolute UV spectrophotometry of $B$ and Be stars. In the range $912 \leq \lambda \leq 3100 \mathrm{~A}$, Brune et a1. (1979) observed $5^{\prime}$ stars including the Be star $\alpha \bar{E} \bar{r} i$. Although $\alpha$ Eri has a stellar wind (Marlborough and Snow, 1976) its spectrum shows Balmer emission only occasionally (Andrews and Breger, 1966). Thus its UV flux deficiency is expected to be sma11, since Beeckmans and Hubert-Delplace (1980) noted that stars with weak Balmer emission had little, if any, UV flux deficit. The absolutely calibrated flux of $\alpha$ Eri agrees well with models for $\lambda>1200 \mathrm{~A}$ and $\mathrm{T}_{\text {eff }}$ obtained by Brune et al. is the same as that of Code et al. (1976). If $\alpha$ Eri is representative of Be stars with no significant UV flux deficit, then Brune et al.'s analysis, together with the conclusions of Nandy and Schmidt (1975) for normal stars of types $\mathrm{B} 0-\mathrm{A} 2$, suggest that theoretical models can reproduce satisfactorily the observed UV fluxes for $\lambda>1200 \mathrm{~A}$. Unfortunately this is not so for $\lambda<1200 \mathrm{~A}$. The best available models do not predict the observed fluxes for $\lambda<1200 \mathrm{~A}$. Even for a star as cool as $\alpha$ Eri the model fluxes are $15 \%$ too large for $912 \leq \lambda \leq 1200 \mathrm{~A}$. This discrepancy increases for hotter stars and appears now tō be a serious problem for 0 stars (Massa and Conti, 1980) and probably for Oe stars as well. Since models overestimate the flux for $912 \leq \lambda \leq 1200$ A by at least $15 \%$, the Lyman continuum fluxes of models are prob̄ably too large as well. Thus quantitative predictions based upon models of the circumstellar matter around Be stars,e.g. Poeckert and Marlborough (1978a), are perhaps even more uncertain than previously thought.

Collins and Sonneborn (1977) have demonstrated how the flux from a star rotating at breakup speed and viewed equator on differs from that of a non-rotator; the rotator can be up to $4^{\mathrm{m}}$ fainter in the UV. This is an extreme example however because there is no evidence that Be stars rotate at breakup speed and because the flux difference increases nonlinearly with rotation rate. Nevertheless differences between rotating and non-rotating stars are expected; it should be recalled however that the quantitative predictions are model dependent. If Be stars are to be used to test these predictions corrections for 1 ine blocking in the $\mathrm{CE}$ and for interstellar extinction must be applied. Heap $(1975,1976)$ compared observations of $\zeta$ Tau to the predictions of Collins (1974) and found agreement could be obtained only if $\zeta$ Tau rotated at breakup speed. There are, however, several problems with her analysis. Because of an erroneous stellar temperature Heap compared the observations to a model of a B1 V star instead of a cooler one. She estimated $\mathrm{E}(\mathrm{B}-\mathrm{V}) \simeq 0.01$; Beeckmans and Hubert-Delplace (1980) 
suggest instead 0.05 to 0.06 . If these latter values are more reliable Heap has underestimated considerably the correction for extinction. Both effects act to increase the difference between the model predictions and the corrected observations of $\zeta$ Tau. Because of problems inherent in correction for line blocking in the CE plus interstellar extinction, it may be prudent to use Be stars like $\alpha$ Eri, which have little UV flux deficiency, or better still rapidly rotating $B$ stars which have never demonstrated Be characteristics.

\section{SPECTRAL CLASSIFICATION AND ANALYSIS OF ULTRAVIOLET SPECTRA}

Numerous investigations support the contention that the physical processes which control the formation of the optical and UV spectra of both $B e$ and $B$ stars of the same spectral type are the same. If however this proves not to be so, then one can assert that the effects of the different processes on optical and UV spectra of Be stars are below the present level of detectability. Cucchiaro et al. (1979 and references therein) have examined many $\mathrm{S} 2 / 68$ spectra of $B$ and $A$ stars and have shown that a self-consistent classification scheme can be produced which correlates well with optical criteria. Peters (1979) has shown that the same physical parameters determine the optical and UV spectra of two Be stars. Brune et a1. (1979) have concluded that the UV energy distribution of $\alpha$ Eri for $\lambda>1200$ A can be well represented by a model whose $T_{\text {eff }}$ is consistent with the optical spectral type. There is no evidence to suggest that the optical spectra of Be stars, which have lost their emission lines, differ in any way from those of rapidly rotating $B$ stars of the same spectral type. Line ratios used to assign optical spectral types for Be stars do not seem to be affected greatly, or perhaps at all, by the $\mathrm{CE}$. Therefore if differences are found between the UV spectra of $B e$ and B stars of the same type, the spectral features which differ are likely to have a circumstellar origin.

Panek and Savage (1976) examined 118 0A0-2 spectra of O,B stars. For dwarfs they discovered that $\lambda 1400$ ( $\mathrm{Si}$ IV) is strongest for types BO-B1 and disappears by B3, while $\lambda 1550$ (C IV) decreases in strength from 0 to $B$ stars and disappears between B2 and B3. For a group of $B e$ stars mostly earlier than B5 they concluded that $\lambda 1400$ was normal in strength but $\lambda 1550$ was stronger than expected in some cases. Note that Barbier and Swings (1979 and references therein) contend that in low resolution spectra cooler than about $B 1$, the major contributor to $\lambda 1550$ is Fe III. Henize et al. (1976) have found $\lambda 1400$ (Si IV) $/ \lambda 1550$ (C IV) to be smaller in Be stars (BO-B2) relative to non-emission $B$ stars; Jaschek (1979) however did not note this on $\mathrm{S} 2 / 68$ spectra. Lamers et a1. (1980a) state that $\lambda 2070$, primarily Fe III, is weaker in Be stars compared to normal $B$ stars and suggest the weakness is due to line emission in the CE. However the lower UV continuum in Be stars with moderate optical emission strength may complicate their interpretation.

These investigations and others considered later demonstrate conclusively that some features in the UV spectra of Be stars differ significantly from normal $B$ stars of the same spectral type. Some of 
these differences are due to the cool circumstellar envelope (CCE), cool in the sense that its kinetic temperature, $\mathrm{T}_{\mathrm{kin}}$, is the same order as $\mathrm{T}_{\text {eff }}$ for the star. Others, like absorption lines of 0 VI $\lambda 1035$, $\mathrm{N} \mathrm{V} \lambda 1240$, C IV $\lambda 1550$ and Si IV $\lambda 1400$, indicate directly the existence of additional sources of ionization and indirectly the presence of hot regions in the CE. Quantitative attempts to interpret these latter as photospheric lines lead to inconsistencies with optical results and as such support one of Jaschek's (1977) arguments for the necessity of an UV classification scheme.

Quantitative investigations of high resolution spectra of a few Be stars have been undertaken. Peters (1979) studied both Copernicus U2 and ground based spectra of $U$ Cyg and $\mu$ Cen and concluded that the observed profiles of UV lines could be fitted with the same model atmosphere, normal abundances and $v$ sin $i$ as determined from the optical spectra. Heap $(1975,1976)$ analysed a high resolution UV spectrum of $\zeta$ Tau and obtained a temperature, significantly higher than that expected from the spectral type, from the Si III/Si IV and C III/C IV ionization balance. The best fits to the observed profiles of $\mathrm{Si}$ and $\mathrm{C}$ lines necessitated abundances $1 / 5$ of solar values and the widths of $C$ IV 1 ines required $\mathrm{v}$ sin $\mathrm{i}<200 \mathrm{~km} \mathrm{~s}^{-1}$, considerably smaller than that from optical spectra. In her analysis Heap assumed that all strong resonance lines in the UV spectrum were photospheric and that the CE would not contribute to lines of high stages of ionization. With regard to $\mathrm{C}$ IV both assumptions are incorrect.

Serious contamination of the $\lambda 1550$ feature by photospheric C IV and $\mathrm{Fe}$ III or by Fe III from the CE can be eliminated. Photospheric $C$ IV is not expected in a star as cool as $\zeta$ Tau and photospheric Fe III is not expected to be strong (Panek and Savage, 1976; Barbier and Swings, 1979). The $\lambda 1930 \mathrm{Fe}$ III feature, which shows a marked increase in strength in supergiants, shell stars and Be stars of large $v$ sin $i$ (Beeckmans, 1975), is observed in $\zeta$ Tau; Heap (1977) has concluded that the individual $\mathrm{Fe}$ III lines at $\lambda 1930$ are formed in the CE. But, as Beeckmans (1975) has emphasized, the lower levels of these Fe III lines are a11 metastable or quasi-metastable, whereas those Fe III 1ines contributing to $\lambda 1550$ have non-metastable lower levels. Thus the relative contribution of Fe III to $\lambda 1930$ and $\lambda 1550$ is expected to be similar to that shown by permitted and forbidden lines in the optical spectra of shell stars (Struve and Wurm, 1938). The lines at $\lambda 1550$ in $\zeta$ Tau are therefore most likely C IV, but contrary to Heap they have a circumstellar origin. Although a re-analysis of Heap's data has not been performed it seems safe to conclude that abundances closer to solar values and a temperature more consistent with the optical spectrum would be obtained.

The low value of $v$ sin $i$ obtained from UV lines (Heap 1975,1976) is a common phenomenon in Be stars of large $v$ sin $i$. In a study of $\zeta$ Oph Morton et a1. (1972) noted that photospheric lines, such as C III $\lambda 1247$, were very narrow and yielded much lower values of $v$ sin $i$.than expected. As one possible explanation they proposed that the narrow 
lines were formed in the more slowly rotating polar regions. In providing the first quantitative explanation Hutchings (1976a) pointed out that gravity darkening due to rapid rotation would produce different continuum brightness distributions in the optical and UV so that the ratio of polar to equatorial flux would increase with decreasing wavelength. For the simple case of a rigidly rotating, undistorted star, which radiates like a black body but suffers gravity darkening according to von Zeipel's law, Hutchings demonstrated that, if each line has a small thermal width relative to rotational broadening, a ratio of the width at half maximum of an idealized line at $\lambda 4500$ to that of one at $\lambda 1000$ as large as 2 could occur for hot stars rotating near breakup speed and viewed at an angle to the rotation axis exceeding $60^{\circ}$. Ratios this large agree with the data of Heap and Morton et al. At viewing angles less than $60^{\circ}$, no significant difference is predicted, just as Peters (1979) observed.

Hutchings et a1. (1979 and references therein) have applied this approach to determine the inclination angle, $i$, to the rotation axis and $\omega / \omega_{c}$, the ratio of angular velocity to the critical value for breakup, for a number of $0, B$ stars including many Be stars. According to their results, Be stars have a random distribution of angles $i$ and a broad distribution of $\omega / \omega$ with a maximum near 0.8 ; significantly Be stars do not as a group rotate at breakup speed.

Because of the importance of these results one must subject the analysis to close scrutiny. Although the conclusions are definitely model dependent the crucial questions to be answered are how sensitive are the predictions to variations of the assumptions used and how important are effects which were not included. Hutchings (1976a) argues that his conclusions are not greatly sensitive to moderate departures from any of his assumptions. On the other hand Sonneborn and Collins (1977) performed similar calculations but used a rotationally distorted star and included the temperature and gravity dependence of actual lines. Quantatively their results resemble Hutchings's but quantitatively their range of half width variation is smaller than both the observations of Heap $(1975,1976)$ and the predictions of Hutchings; they also note a strong dependence on the choice of basic stellar parameters.

Many other factors may influence Hutchings's conclusions. These include the problem of differential versus rigid rotation and errors in the estimate of the breakup speed from uncertainties in the equatorial radius of the distorted star and in the mass, if estimated from the spectral type (Slettebak et al., 1980). Finally evidence exists to suggest that some $B$ and $B e$ stars have dynamic and/or unstable photospheres. This evidence includes: stellar winds in Be stars (discussed below); radial motions in the photosphere of $\tau$ Sco (Smith and Karp, 1979); and $\beta$-Cephei-type variations in the Be stars $\omega$ CMa (Baade,1979) and 19 Mon (Balona and Engelbrecht, 1979). If such phenomena are common the line of sight velocity component of a photospheric mass element may contain macroscopic velocity components in addition to rotational ones. Recently Mihalas (1979) has shown that atmospheric velocity fields can 
produce significant centre to limb variations of profiles in expanding atmospheres. The assumption of a unique intrinsic profile with which to deduce $v$ sin $i$ from observed line widths is thus highly questionable. For $\zeta$ Oph, Hutchings and Stoeckley (1977) derive $i=55^{\circ}$, rotation speed $=475 \mathrm{~km} \mathrm{~s}^{-1}$ giving $\mathrm{v}$ sin $i=390 \mathrm{~km} \mathrm{~s}^{-1}$, and $\omega / \omega_{\mathrm{c}}=0.85$ for a breakup speed of $560 \mathrm{~km} \mathrm{~s}^{-1}$. However Walker et al. (1979) observed the variation of distinctive features of the He I $\lambda 6678$ 1ine, which they presumed to arise from a non uniform surface brightness distribution, and derived either $\mathrm{v}$ sin $i=560 \mathrm{~km} \mathrm{~s}^{-1}$, if these non uniformities are equatorial, or $v$ sin $i=508 \mathrm{~km} \mathrm{~s}^{-1}$ using $i=55^{\circ}$ from Hutchings and Stoeckley. In either case their estimate of $v$ sin $i$ differs greatly from Hutchings and Stoeckley.

Therefore there are numerous effects which may be important but were not included by Hutchings (1976a). Whether inclusion of any of these will lead to different predictions is unknown. This problem has many similarities to the LTE, non-LTE debate of a decade or more ago. Perhaps the only way to convince oneself that Hutchings's approach is satisfactory is to redo the analysis including as many of the above effects as possible. Until this is done all one can do is to emphasize again the warning expressed by both Mihalas and Sonneborn and Collins that properties of rotating stars determined from observational data are model dependent.

\section{THE COOL CIRCUMSTELLAR ENVELOPE}

In the past the CCE has been investigated primarily at optical and infrared wavelengths. Although reliable estimates of its $\mathrm{T}_{\mathrm{kin}}$ are difficult to obtain, $\mathrm{T}_{\mathrm{k}}$ in $\sim 10^{4} \mathrm{~K}$ is consistent with photoionization by Lyman continuum radiation, with infrared excesses due to free-bound and free-free emission (Gehrz et al., 1974) and with the presence of singly and doubly ionized ions in shell star spectra. Various arguments suggest the CCE is more disk-like than spherically symmetric (Marlborough, 1976). Disk-like however does not mean a disk of constant thickness since the scale height of the CCE perpendicular to the equatorial plane is expected to increase with distance from the rotation axis.

Because the UV flux deficiency seems to increase with increasing envelope density and because one of the contributors to absorption at $\lambda 2100$ are Fe III transitions whose lower levels are metastable (Beeckmans, 1975), a Be star with a small but significant UV deficit could be called a shell star even though its optical spectrum shows no shell lines. Examples of such cases, where individual Fe III resonance 1 ines are present in Copernicus spectra, were noted by Snow et al. (1979). Perhaps then, as Hutchings (1976b) suggested, shell stars are simply Be stars in which the CCE has a larger average density and/or is much more extensive than average.

If all Be stars are rapid rotators and if the CCE is concentrated to the equatorial plane one might expect to discover a dependence of 
Fe III line strength and UV flux deficiency on $v$ sin $i$. However other factors such as envelope density, ionization structure and extent of the $C E$ perpendicular to the equatorial plane will also influence Fe III line strength, so it is perhaps not surprising that Snow et a1. (1979) found only a weak dependence on $v$ sin $i$. Beeckmans and Hubert-Delplace (1980) found no pole-on Be stars to have a statistically significant UV deficit; nor does $X$ Oph even though it has very strong Balmer emission and an infrared excess. Since these stars have smaller than average $v$ sin $i$ the lack of a UV deficiency may support weakly a nonspherical CCE.

The radial velocities of 1 ines of various stages of ionization led Snow et al. (1979) to conclude that both ionization and expansion velocity of the CCE increase outward. Both Marlborough (1977) and Snow et al. have suggested that all Be stars have stellar winds which have low expansion velocity near the star and degree of asymmetry determined by factors peculiar to each star. The CCE is then the deeper, denser and more slowly moving region of such a wind.

\section{ANOMALOUS STAGES OF IONIZATION AND THE HOT CIRCUMSTELLAR ENVELOPE}

One of the interesting and important results of UV observations of $O, B$ and $B e$ stars was the discovery of lines from stages of ionization, especially $0 \mathrm{VI}$ and $\mathrm{N} \mathrm{V}$ but also $\mathrm{C}$ IV and $\mathrm{Si}$ IV in B stars, whose photospheric number densities had been expected to be insignificant were photospheric radiation the only ionizing source. These ions must have an origin in the $\mathrm{CE}$ and their presence requires additional sources of ionization (Conti and de Loore, 1979). In main sequence stars and $\mathrm{Be}$ stars the resonance lines from these stages of ionization are usually absorption lines.

Lamers and Snow (1978) considered the ionization conditions in the $\mathrm{CE}$ of $\mathrm{O}, \mathrm{B}$ stars, assuming the ionization source was dilute photospheric radiation, and concluded that in main sequence stars one should not see $0 \mathrm{VI}$ at all, $\mathrm{N} \mathrm{V}$ in stars with $\mathrm{T}_{\text {eff }} \lesssim 40,000 \mathrm{~K}(\sim 07)$, and $\mathrm{Si}$ IV in stars with $\mathrm{T}_{\text {eff }} \lesssim 22,000 \mathrm{~K}(\sim \mathrm{B} 3)$. By interpolation using ionization potentials C IV would not be expected in dwarfs with $\mathrm{T}_{\text {eff }} \leqslant 30,000$ $\mathrm{K}(\sim \mathrm{B1})$. Kamp's (1978) results for the Si spectra in B stars agree with Lamers and Snow. Studies of high and low resolution UV spectra of normal dwarfs by Panek and Savage (1976), Barbier and Swings (1979) and Upson and Rogerson (1980) confirm respectively the absence of photospheric C IV in stars cooler than about BI and photospheric Si IV cooler than about B3. Snow and Morton (1976) and Rogerson and Upson (1977) note that $O \mathrm{VI}$ and $\mathrm{N} \mathrm{V}$ are present in dwarfs hotter than $\mathrm{B1}$; in normal dwarfs cooler than B1 there is no evidence for either ion. Therefore normal main sequence stars cooler than B2-B3 do not show any evidence in their UV spectra for anomalous stages of ionization.

For Be stars, O VI is definitely present at 09 (Morton, 1979) but 
not confirmed in any cooler Be star. Lines of $\mathrm{N} \mathrm{V}$ have been reported in Be stars hotter than about B1 (Morton, 1976; Marlborough, 1977), but not detected at B2 (Peters, 1979). Lines of Si IV occur in stars as cool as B5 (Lamers and Snow, 1978). Recent results indicate that both C IV and Si IV are present at B5 and Si IV probably in stars as coo1 as B8 (Marlborough and Peters, 1982). These results are summarized in Figure 1 .

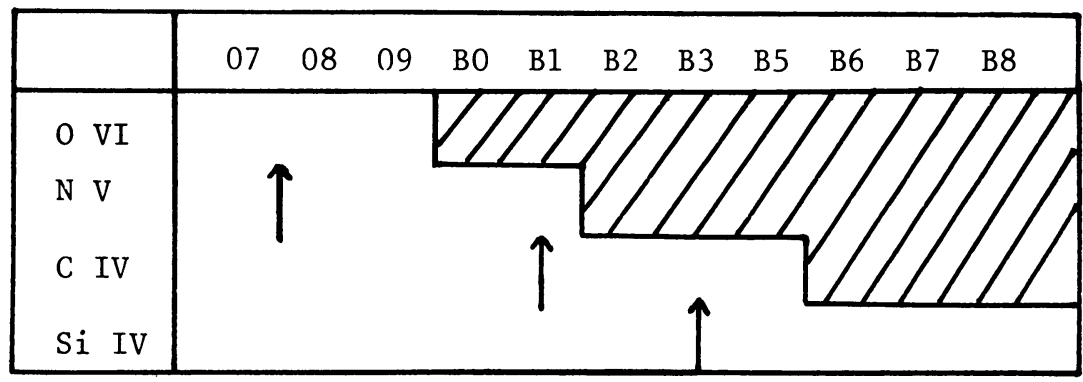

Figure 1. Distribution of anomalous stages of ionization in Be stars. The hatched region shows the range in which the ion is not detected in Be stars; the vertical arrow, the coolest spectral type for the ion to be present in normal dwarfs.

Therefore, UV spectra covering the entire range of Be stars show the presence of ions whose existence requires ionization sources in addition to photospheric radiation. If this additional source is collisional ionization, the $\mathrm{CE}$ must contain regions in which $\mathrm{T}_{\mathrm{k}}$ in varies from $\sim 3 \times 10^{5} \mathrm{~K}$ in 1 ate 0 and early $\mathrm{B}$ stars showing $0 \mathrm{VI}$ and $\mathrm{N}$, to $\sim 7 \times 10^{4} \mathrm{~K}$ in late B stars showing $\mathrm{C}$ IV and Si IV (Lamers and Snow, 1978).

If the ionization is radiative some part of the $\mathrm{CE}$ must have $\mathrm{T}_{\mathrm{kin}} \gtrsim 10^{6} \mathrm{~K}$ to produce sufficient soft x-rays by bremsstrahlung (Cassinellil and 01son, 1979). In either case the CE contains hot regions (HCE) in addition to cool ones.

\section{VARIABILITY OF ULTRAVIOLET SPECTRA}

Although few systematic searches for variations have been attempted numerous reports document changes on time scales from hours to years. Changes in the strength of Si III $\lambda \lambda 1388$ and 1500 were detected by Hammerschlag-Hensberge et al. (1980) in $\gamma$ Cas over about 4 weeks. These are probably real photospheric variations because, although $\lambda 1388$ could be affected by narrow, highly shifted components of Si IV $\lambda 1394$ (Henrichs et al., 1980), there is no obvious contaminant for $\lambda 1500$ and because the excitation energy of the common lower level of both transitions is $17.7 \mathrm{ev}$. 
Variability of spectral features ascribed to the CCE has been reported. Dramatic changes in the UV spectrum of 59 Cyg, concurrent with the formation and dissipation of optical she11s, were discussed by Beeckmans (1976) and Marlborough and Snow (1980). Marlborough et al. (1978) noted changes over two years in the emission components of $\mathrm{Mg}$ II $\lambda 2800$ in the $\gamma$ Cas; corresponding changes in Si IV core emission also seemed to occur (Slettebak and Snow, 1978). During simultaneous UV and optical observations of $\gamma$ Cas, Slettebak and Snow (1978) reported that a sudden change in $\mathrm{H} \alpha$ in a few hours seemed to be accompanied by an increase in Mg II emission and perhaps also in Si IV core emission.

Much data exist demonstrating variations in the HCE for the hotter Be stars. Large changes have occurred in the region around $\lambda$ 1240: Marlborough and Snow (1980) and Doazan et al. (1980) discuss changes in N V in 59 Cyg over a period of years; Marlborough et al. (1978) show evidence for dramatic variations in $\gamma$ Cas at $\lambda 1240$ in two hours. Modest C IV emission in $\gamma$ Cas, noted by Bohlin (1970), was not detected later by Panek and Savage (1976) nor by Marlborough and Peters (1982). Panek and Savage did find significant variations in C IV and $\mathrm{Si}$ IV absorption in $\gamma$ Cas over one year. Narrow absorption components in each of $\mathrm{N} \mathrm{V}, \mathrm{C} \mathrm{IV}$ and $\mathrm{Si}$ IV in $\gamma \mathrm{Cas}$, which appear in a time as short as a week and disappear within 2-3 months, were discovered by Henrichs et a1. (1980). Features of this kind occur commonly in 0,B star winds (Lamers et al., 1980b).

The UV variations that have been observed in the CCE seem to be analogous to the well-known optical variations, especially those of longer time scale. The observed changes in the HCE may be more closely related to those in the winds of $0, B$ stars (Snow, 1979). Clearly systematic studies need to be undertaken to determine whether variations are common to Be stars of all spectral types and to discover the true time scales of variability.

\section{STELLAR WINDS AND MASS LOSS}

Radial velocities exceeding the escape speed in the region where the lines arise are generally considered to be direct evidence for mass loss. Mass loss and associated stellar winds have long been suspected in Be stars but never demonstrated conclusively until the advent of UV observations. UV data which have been used to infer or demonstrate mass loss are summarised in Table 2. Column 3 contains the quoted radial velocity or the most negative value listed if several stars were discussed. For main sequence stars of types 05,B0,B5 and A0, upper 1imits to the escape speed from the surface are respectively $920,960,800$ and $700 \mathrm{~km} \mathrm{~s}-1$ (Allen, 1973). Clearly the absolute values of many of the entries exceed these and thus provide evidence for mass loss. Note however that radial velocities exceeding the escape speed are obtained primarily from constituents of the HCE. 
TABLE 2

Ultraviolet Features Indicating Winds and Mass Loss in Be Stars.

Star or Type UV Features Observed $\begin{aligned} & \mathrm{RV}_{-1} \\ & \text { of Star }\end{aligned}$
REFERENCE

\begin{tabular}{|c|c|c|c|}
\hline$\gamma$ Cas & C IV & $<-450$ & Bohlin (1970) \\
\hline$\zeta$ Oph & C IV & -1300 & Smith and Stecher (1971) \\
\hline$\zeta \mathrm{Oph}$ & C IV, core & -900 & Morton et a1.(1972) \\
\hline$\zeta$ Tau & UV resonance lines & -120 & Heap (1975) \\
\hline $\begin{array}{l}\mathrm{B} 0-\mathrm{B} 5, \text { large } \\
\mathrm{v} \sin \mathrm{i}\end{array}$ & Si IV, short $\lambda$ edge & $\sim-1000$ & $\begin{array}{l}\text { Snow and Marlborough(1976), } \\
\text { Lamers and Snow(1978) }\end{array}$ \\
\hline$\eta$ Cen & Si IV, short $\lambda$ edge & -250 & Burton and Evans (1976) \\
\hline $\begin{array}{l}\mathrm{B} 0-\mathrm{B} 3,1 \text { arge } \\
\mathrm{v} \sin \mathrm{i}\end{array}$ & resonance line cores & -240 & Marlborough (1977) \\
\hline$\gamma$ Cas & Mg II, core & -215 & Morgan et al. (1977) \\
\hline$\phi$ Per & $\mathrm{Fe}$ III, $\lambda 2062,2068,2079$ & -350 & Bruhweiler et a1.(1977) \\
\hline 59 Cyg & $\mathrm{N} V$, short $\lambda$ edge & $<-830$ & Marlborough and Snow(1980) \\
\hline 59 Cyg & $\mathrm{N}$ V,C IV, cores & -750 & Doazan et al. (1980) \\
\hline$\curlyvee$ Cas & $\mathrm{N}$ V,C IV,Si IV components & -1500 & Henrichs et a1.(1980) \\
\hline $\begin{array}{l}\gamma \text { Cas, X Per } \\
\text { HD102567 }\end{array}$ & Si IV, short $\lambda$ edge & $\begin{array}{l}-800 \text { to } \\
-1000\end{array}$ & $\begin{array}{l}\text { Hammerschlag-Hensberge } \\
\text { et al. (1980) }\end{array}$ \\
\hline
\end{tabular}

Details of the determination of mass loss rates together with recent results are given by Snow (1982).

ACKNOWLEDGEMENT

I wish to thank Professor R.J. Tayler and the staff of the Astronomy Centre, University of Sussex, for their hospitality while this review was prepared. I have benefited greatly from discussions with friends and colleagues, especially P.K. Barker, G.W. Collins, D.G. Hummer, L. Meste1, G.J. Peters, R. Poeckert, R.C. Smith and T.P. Snow. I also wish to thank M. Rasche for her assistance. This work was supported by the Science Research Council (UK) and the Natural Science and Engineering Research Council (Canada). 


\section{REFERENCES}

Aalders, J.W.G., van Duinen, R.J., Luinge, W., and Wildemann, K.J.:

1975, Space Sci. Instrumentation 1, pp. 343-350.

Abbott, D.C., Bieging, J.H., Churchwe11, E., and Cassine11i, J.P.: 1980, Astrophys. J. 238,pp. 196-202.

Allen, C.W.: 1973, 'Astrophysical Quantities', Athlone Press, London.

Andrews, P.J., and Breger, M.: 1966, Observatory 86,pp.108-109.

Baade,D.: 1979, ESO Messenger, December, pp.4-6.

Balona, L.A., and Engelbrecht, C.: 1979, Monthly Notices Roy.Astron.Soc. 189,pp.171-174.

Barbier,R., and Swings, J.P.: 1979, Astron. Astrophys . 72,pp.374-375.

Beeckmans, F.: 1975, Astron.Astrophys . 45,pp.177-183.

Beeckmans, F.: 1976, Astron.Astrophys. 52,pp.465-466.

Beeckmans, F., and Hubert-Delplace, A.M.: 1980, Astron.Astrophys. 86, pp. 72-86.

Boggess, A., Carr, F.A., Evans, D.C., Fische1, D., Freeman, H.R., Fuechsel, C.F., Klinglesmith, D.A.,Krueger, V.L., Longanecker, G.W., Moore, J.V., Pyle,E.J., Rebar, F., Sizemore, K.0., Sparks, W., Underhil1, A.B., Vitagliano, H.D., West, D.K., Macchetto, F., Fitton, B., Barker, P.J., Dunford, E., Gondhalekar, P.M., Ha11, J.E., Harrison, V.A.W., Oliver, M.B., Sandford, M.C.W., Vaughan, P.A., Ward, A.K., Anderson, B.E., Boksenberg, A., Coleman, C.I., Snijders, M.A.J., and Wilson, R.: 1978, Nature 275, pp.2-7.

Bohlin, R.C.: 1970, Astrophys. J. 162,pp.571-587.

Boksenberg, A., Evans, R.G., Fowler, R.G., Gardner, I.S.K., Houziaux, L., Humphries, C.M., Jamar, C., Macau,D., Macau, J.P., Malaise,D., Monfils,A., Nandy, K., Thompson, G.I., Wilson R., and Wroe, H.: 1973, Monthly Notices Roy.Astron.Soc. 163,pp.291-322.

Briot, D.: 1978, Astron.Astrophys. 66,pp.197-203.

Bruhweiler, F.C., Morgan, T.H., and van der Hucht, K.A.: 1978, Astrophys.J.Letters 225,pp.L71-L74.

Brune, W.H., Mount, G.H., and Feldman, P.D.: 1979, Astrophys.J. 227 , pp. 884-899.

Burger, M., and van der Hucht, K.A.: 1976, Astron.Astrophys. 48,pp.173185.

Burton, W.M., and Evans, R.G.: 1976, in A.Slettebak (ed.), 'Be and Shell Stars, IAU Symp. 70',pp.199-207.

Cassine11i, J.P., and 01son, G.L.: 1979, Astrophys.J. 229,pp.304-317.

Code, A., Houck, T.E., McNa11, J.F., Bless, R.C., and Lillie, C.F.: 1970, Astrophys.J. 161,pp. 377-388.

Code, A., Davis, J., Bless, R.C., and Hanbury Brown, R.: 1976, Astrophys. J. $203, \mathrm{pp} .417-434$,

Collins, G.W.: 1974, Astrophys.J. 191,pp.157-164.

Collins, G.W., and Sonneborn, G.: 1977, Astrophys.J.Supp1. 34, pp.41-94.

Conti, P.S., and de Loore, C.B. (eds).: 1979, 'Mass Loss and Evolution of 0-Type Stars, IAU Symp. 83', pp.169-234.

Cucchiaro, A., Macau-Hercot, D., Jaschek, M., and Jaschek,C.: 1979, Astron.Astrophys.Supp1. 35,pp.75-82.

Davis, R.J., Deutschman, W.A., Lundquist, C.A., Nozawa, Y., and Bass, S.D.: 1972, in A.Code (ed,) 'The Scientific Results from the Orbiting Astronomical Observatory', NASA SP-310, Washington,pp.1-22. 
Doazan, V., Kuhi, L.V., and Thomas, R.N.: 1980, Astrophys,J.Letters 235, pp. L17-L20.

Frost, S.A., and Conti, P.S.: 1976, in A. Slettebak (ed.), 'Be and Shell Stars, IAU Symp. 70',pp.139-147.

Gehrz, R.D., Hackwe11, J.A., and Jones, T.W.: 1974, Astrophys,J. 191, pp. $675-684$.

Gurzadyan, G.A.: 1975, Space Sci.Rev. 18,pp.95-139.

Hammerschlag-Hensberge, G., van den Heuve1, E.P.J., Lamers, H.J.G.L.M., Burger,M., de Loore,C., Glencross,W., Howarth,I., Willis,A.J., Wilson,R., Menzies,J., Whitelock,P.A., van Dessel,E.L., and Sandford,P.: 1980,Astron.Astrophys. 85,pp.119-127.

Heap, S.R.: 1975, Phil.Trans.Roy.Soc.London A 279,pp.371-377.

Heap, S.R.: 1976, in A.Slettebak (ed.) 'Be and Shell Stars, IAU Symp. 70', pp. 165-178.

Heap, S.R.: 1977, Astrophys.J. 217,pp.90-94.

Henize, K.G., Wray,J.D., Parsons, S.B., and Benedict,G.F.: 1976, in A Slettebak (ed.) 'Be and Shel1 Stars, IAU Symp. 70',pp.191-195.

Henrichs,H.F., Hammerschlag-Hensberge,G., and Lamers,H.J.G.L.M.: 1980, paper presented at Second European IUE Conference.

Henry,R.C., Weinstein,A., Feldman,P.D., Fastie,W.G., and Moos,H.W.: 1975, Astrophys.J. 201,pp.613-623.

Hutchings,J.B.: 1976a, Pub.Astron.Soc. Pacific 88, pp.5-7.

Hutchings, J.B.: 1976b, in A.Slettebak(ed.), 'Be and Shell Stars, IAU Symp. 70',pp.13-27.

Hutchings, J.B., and Stoeckley,T.R.: 1977, Pub.Astron.Soc. Pacific 89,pp.19-22.

Hutchings, J.B., Nemec,J.M., and Cassidy. J.: 1979, Pub. Astron.Soc. Pacific 91,pp. 313-318.

de Jager, C., Hoekstra,R., van der Hucht,K.A., Kamperman,T.M., Lamers, H.J., Hammerschlag,A., Werner,W., and Emming,J.G.: 1974, Astrophys. Space.Sci. 26,pp.207-262.

Jaschek,C.: 1977, in E. Muller (ed.), 'Highlights of Astronomy', 4 part II, pp. 283-288.

Jaschek,C.: 1979, quoted by A.Slettebak: 1979,Space Sci.Rev. 23,pp.541 -580 .

Kamp,L.: 1978,Astrophys.J.Supp1. 36,pp.143-171.

Kondo,Y., Modisette,J.L., and Wolf,G.W.: 1975, Astrophys.J. 199, pp.110-119.

Kondo,Y., de Jager,C., Hoekstra, R., van der Hucht,K,A., Kamperman,T.M., Lamers, H.J.G.L.M., Modisette,J.L., and Morgan, T.H.: 1979, Astrophys.J. 230,pp.526-533.

Kurucz,R.L.: 1974, Astrophys.J. Letters 188, pp.L21-L22.

Lamers, H.J.G.L.M., and Rogerson,J.B.: 1978, Astron.Astrophys. 66, pp. 417-430.

Lamers,H.J.G.L.M., and Snow,T.P.: 1978, Astrophys.J. 219,pp.504-514.

Lamers,H.J.G.L.M., Faraggiana,R., and Burger,M.: 1980a,Astron.

Astrophys. 82. pp.48-52.

Lamers,H.J.G.L.M., Gathier,R., and Snow,T.P.: 1980b, preprint.

Lillie,C.F., Bohlin,R.C., Molnar,M.R., Barth,C.A., and Lane,A.L.: 1972, Science 175, pp.321-322.

McLaughlin,D.B.: 1961, J.Roy.Ast.Soc.Can. 55,pp. 13-22 and pp.73-85. 
Marlborough, J.M.: 1976, in A.Slettebak (ed.), 'Be and Shell Stars, IAU Symp. 70', pp. 335-370.

Marlborough, J.M.: 1977, Astrophys.J. 216, pp.446-456.

Marlborough, J.M., and Snow,T.P.: 1976, in A.Slettebak (ed.), 'Be and Shell Stars, IAU Symp. 70', pp. 179-189.

Marlborough, J.M., Snow,T.P., and Slettebak, A.: 1978, Astrophys.J. 224 , pp.157-166.

Marlborough, J.M., and Snow,T.P.: 1980, Astrophys.J. 235,pp. 85-96.

Marlborough, J.M. and Peters, G.J.: 1982 in M. Jaschek and H.G. Groth (eds.), 'Be Stars', IAU Symp. 98, pp. 387-390.

Massa,D., and Conti, P.S.: 1980, preprint.

Mihalas,D.: 1979, Monthly Notices Roy.Astron.Soc. 189,pp.671-699.

Morgan, T.H., Kondo,Y., and Modisette, J.L.: 1977, Astrophys.J. 216, pp.457-461.

Morton, D.C.: 1976, Astrophys.J. 203,pp.386-398.

Morton, D.C.: 1979, Month1y Notices Roy.Astron.Soc. 189,pp. 57-68.

Morton, D.C., Jenkins, E.B., Matilsky, T.A., and York, D.G.: 1972, Astrophys.J. 177, pp.219-234.

Nandy, K., and Schmidt,E.G.: 1975, Astrophys.J. 198, pp.119-125.

O'Callaghan, F.G., Henize, K.G., and Wray, J.D.: 1977, Applied Optics 16, pp.973-977.

Panek, R.J., and Savage, B.D.: 1976, Astrophys.J. 206,pp. 167-181.

Peters, G.J.: 1979, Astrophys.J.Supp1. 39, pp.175-193.

Peytremann, E.: 1975, Astron.Astrophys. 39,pp. 393-403.

Poeckert,R., and Marlborough,J.M.: 1978a Astrophys.J. 220,pp.940-961.

Poeckert, R., and Mar1borough, J.M.: 1978b, Astrophys.J.Suppl.

38 , pp. 229-252.

Rogerson, J.B., Spitzer, L., Drake, J.F., Dressler, K., Jenkins, E.B., Morton,D.C., and York,D.G.: 1973, Astrophys.J.Letters 181, Pp.L97-L102.

Rogerson, J.B., and Upson, W.L.: 1977, Astrophys.J.Supp1. 35,pp.37-110.

Slettebak, A., and Snow, T.P.: 1978, Astrophys.J.Letters 224, pp.L127-L131.

Slettebak, A., Kuzma, T.J., and Collins, G.W.: 1980,Astrophys.J. 242, pp. 171-187.

Smith, A.: 1976, Astrophys.J. 147, pp.158-171.

Smith, A., and Stecher, T.P.: 1971, Astrophys.J.Letters 164,pp.L43-L47.

Smith, M.A., and Karp, A.H.: 1979,Astrophys.J. 230,pp.156-161.

Snow, T.P.: 1979, in P.Conti and C.de Loore (eds.) 'Mass Loss and Evolution of 0-Type Stars, IAU Symp. 83' pp.65-80.

Snow, T.P.: 1982, in M. Jaschek and H.G. Groth (eds.), 'Be Stars', IAU Symp. 98, pp. 377-385.

Snow, T.P., and Morton, D.C.: 1976, Astrophys.J.Supp1. 32,pp.429-465.

Snow, T.P., and Marlborough, J.M.: 1976, Astrophys.J.Letters 203, PP.L87-L90.

Snow, T.P., Peters, G.J., and Mathieu, R.D.: 1979, Astrophys.J.Supp1. 39,pp.359-376.

Sonneborn, G.H., and Collins, G.W.: 1977, Astrophys.J. 213,pp.787-790.

Struve,0., and Wurm,K.: 1938, Astrophys.J. 88, pp.84-109.

Troy,B.E., Johnson, C.Y., Young, J.M., and Holmes, J.C.: 1975, Astrophys.J. 195, pp. 643-648.

Upson, W.L., and Rogerson, J.B.: 1980, Astrophys.J.Supp1. 42, pp.175-220. 
Walker, G.A.H., Yang, S. and Fahlman, G.G.: 1979, Astrophys. J., 233, pp. 199-204.

\section{DISCUSSION}

Doazan: I would like to comment on the UV "deficiencies" found by Beekmans and Hubert-Delplace that you report. To accomplish the comparison of UV fluxes of Be stars relative to normal B stars, one has to solve two separate problems. First, one has to find the correlation of the interstellar extinction, because $\mathrm{E}(\mathrm{B}-\mathrm{V})$ is contaminated by intrinsic reddening. Second, one has to place the Be and B stars at the same distance. If one normalizes the fluxes of $B$ and $B e$ stars at the same magnitude $V$, one will systematically underestimate the UV flux of the Be stars relative to the B stars because, as is well known, Be stars are more luminous than B stars in the visual. The "deficiency" obtained by the authors points toward the direction of the expected results. So, the only conclusion the authors can derive is that the colours of Be stars are redder than those of normal B's. In no way the method used in this study allows to conclude anything about the "excesses" or "deficiencies" of the UV fluxes of Be stars relative to B's.

Paterson-Beekmans: In the work referenced, we have never considered "absolute flux" deficiencies, but always UV fluxes relative to the visible fluxes (in the $\mathrm{V}$ band).

Slettebak: With regard to the spectral type of $\zeta$ Tau from optical spectra, I believe that the value you quote is too large. Morgan classified this star as B1IV (it appears in the Morgan - Abt Tapscott spectral atlas of stars earlier than the sun) and I confirm this type in my recent classification of all the Be stars brighter than magnitude 6.0 .

Sonneborn: First, the point of studying the properties of normal B stars is very important. We should first determine the differences (observationally and theoretically) in the UV spectrum between rotating and nonrotating normal $B$ stars in order to help sort out the effects of a circumstellar envelope. With respect to the UV energy distributions, the models published in 1977 did not include the effects of metallic line blanketing. Our new grid of models do include line blanketing and therefore provide more realistic models of the UV energy distribution in rotating stars. These models will be published soon.

Poeckert: I would like to comment that winds and highly ionized species are not found in SMC OB stars, according to Hutchings (Ap.J. 237, 285, 1980; paper in preparation 1981). Thus it appears that the presence of coronae may be related to metal abundances. 